\title{
Deserción y reprobación, desde el enfoque del estudiantado en la educación superior, en el curso de Matemática General ${ }^{1}$
}

\begin{abstract}
Por: M.Sc. Randall Hidalgo Mora ${ }^{2}$, Universidad Nacional de Costa Rica, Costa Rica, ORCID: 0000-0002-8405-1600, Dr. Ronny Gamboa Araya ${ }^{3}$, Universidad Nacional de Costa Rica, Costa Rica, ORCID: 0000-00029531-0372 y Dr. Mario Castillo Sánchez ${ }^{4}$, Universidad Nacional de Costa Rica, Costa Rica, ORCID: 0000-0002-2078-0628
\end{abstract}

Recibido: 26 de marzo, 2019.

Aprobado: 21 de noviembre, 2019.

\section{RESUMEN}

Este artículo describe la deserción y reprobación en el primer curso de Matemática en la Universidad Nacional de Costa Rica desde el enfoque del estudiante y traza líneas generales en cuanto a posibles causas. Este curso presenta altos índices de deserción y reprobación que repercuten en otras problemáticas, como el avance académico del estudiante. Además, genera implicaciones individuales y sociales en términos de las expectativas estudiantiles, familiares y emocionales por la discrepancia entre las ambiciones de los jóvenes y sus logros, así como consecuencias económicas para el sistema universitario.

En esta investigación se trabajó con toda la población de estudiantes matriculados en el I y II ciclo del año 2017. A ellos se les aplicó un cuestionario, vía correo electrónico, en el cual se les consultó sobre aspectos generales y posibles causas de deserción y reprobación.

El análisis arrojó los siguientes resultados: un alto porcentaje de deserción, independientemente del ciclo lectivo; un mayor porcentaje de aprobación durante el II ciclo, especialmente en las mujeres, posiblemente asociado a un proceso de aprendizaje o de adaptación a la vida universitaria; que la mayoría de los estudiantes proviene de colegios públicos y presenta mayor porcentaje de reprobación y que las causas de deserción priorizadas por los estudiantes son el bajo rendimiento académico, los conocimientos previos y los hábitos de estudio.

Al respecto, puede concluirse que resulta necesario generar políticas institucionales con las cuales sea posible disminuir la deserción y reprobación, específicamente en cursos de primer ingreso a la universidad y, por tanto, acortar tiempos de graduación y optimizar recursos institucionales.

1 La presente investigación se enmarca dentro del proyecto denominado "La deserción y repitencia en el curso de Matemática General en la Universidad Nacional”, código SIA 0250-15, Universidad Nacional, Costa Rica.

2 Máster en Política Económica, Universidad Nacional y licenciado en la Enseñanza de la Matemática por la Universidad Nacional de Costa Rica. Labora como académico de la Escuela de Matemática de la Universidad Nacional de Costa Rica. Contacto: randall.hidalgo.mora@una.ac.cr.

3 Doctor en Educación de la Universidad Estatal a Distancia de Costa Rica y máster en Matemática Educativa por el Centro de Investigación y Estudios Avanzados del Instituto Politécnico Nacional, México. Se desempeña en la Escuela de Matemática de la Universidad Nacional de Costa Rica. Contacto: ronny.gamboa.araya@una.ac.cr.

4 Doctor en Educación de la Universidad Estatal a Distancia de Costa Rica y máster en Estadística, por la Universidad de Costa Rica. Es investigador y académico de la Escuela de Matemática de la Universidad Nacional de Costa Rica. Contacto: mario.castillo.sanchez@una.ac.cr.
Randall Hidalgo Mora, Ronny Gamboa Araya y Mario Castillo Sánchez. Deserción y reprobación, desde el enfoque del estudiantado en la educación superior, en el curso de Matemática General. Revista Comunicación. Año 40, volumen 28, número 2, julio-diciembre, 2019. Instituto Tecnológico de Costa Rica. ISSN: 0379-3974 / e-ISSN1659-3820.

\section{PALABRAS CLAVE:}

Deserción escolar, fracaso escolar, rendimiento académico, matemáticas, educación universitaria, estudiantes.

\section{KEY WORDS:}

Desertion, academic failure, student achievement, mathematics, university education, students. 


\section{ABSTRACT}

\section{Desertion and failure, according to university students, taking General Mathematics}

This article describes desertion and failure in the first course of Mathematics at the National University of Costa Rica from the students' point of view and draws general lines regarding possible causes.

This course has high desertion and failure rates that affect academic progress, as it has both, personal and social implications in terms of student and family expectations: emotional effects due to the discrepancy between the students' aspirations and their achievements; and economic consequences for the university system.

This research surveyed the whole population of students enrolled in the first and second school year of 2017 . Students filled out a questionnaire via email addressing general aspects and possible causes of desertion and failure.

The analysis showed a high desertion rate regardless of the school period; the highest percentage of approval was in the second cycle, especially in women, possibly associated with a learning process or adaptation to university life. Students that constitute the lower percentage of approval and higher percentage of failure come from public schools, which happens to make up the majority of the university population. Students point to low academic performance, previous knowledge, and study habits as the causes of desertion.

It is necessary to generate institutional policies that reduce the desertion and disapproval, specifically in courses of first entrance to the university, and therefore shorten graduation times and optimize institutional resources.

\section{INTRODUCCIÓN}

El propósito de este artículo es mostrar una descripción de la deserción y reprobación en el primer curso de Matemática General en la Universidad Nacional (UNA), Costa Rica, desde el enfoque del estudiante, así como trazar líneas generales en cuanto a las posibles causas de estos indicadores. Para los docentes y estudiantes, resulta preocupante el progresivo aumento que ha tenido la reprobación y la deserción en los cursos del área de Matemáticas, a nivel universitario. También existe un alto grado de abandono de cursos, de ausentismo y una postergación de la graduación más allá de lo esperado. Al respecto González (2007) indica que solo alrededor del 10\% de los estudiantes admitidos en la Universidad de Costa Rica se gradúa en el tiempo establecido por los planes de estudio.

De acuerdo con Torres, Acevedo y Gallo (2015) y Rodríguez (2017), los niveles de deserción y de reprobación son retos e indicadores indirectos de la calidad de los sistemas educativos, entre los cuales se incluye la educación universitaria; sus niveles y sus causas (al ser una parte del fracaso escolar universitario) es un tema que tiene que ser abordado para una correcta planificación de los procesos educativos.
A nivel de educación superior, es una problemática que debe ser atendida desde diferentes instancias universitarias.

Por tal motivo, resulta necesario generar políticas institucionales o propias de las unidades académicas, que permitan disminuir la deserción y reprobación, específicamente en cursos de primer ingreso a la universidad, y, por tanto, acortando los tiempos de graduación y optimizar los recursos institucionales.

\section{LA DESERCIÓN Y REPROBACIÓN EN LA EDUCACIÓN UNIVERSITARIA}

La problemática del bajo rendimiento académico, la reprobación, la deserción y la interrupción en el estudio han sido descritas, generalmente, en tasas brutas; otros estudios han incorporado aspectos como caracterización de factores sociales, regionales e institucionales del estudiantado, así como la perspectiva que este tiene sobre esta problemática.

También el tema de la deserción universitaria es un fenómeno que se ha estudiado desde diferentes enfoques a nivel mundial. Rodríguez y Vindas (2005), por ejemplo, estudian la magnitud del fenómeno, así como las características generales que identifican a 
dicha población, y señalan que existen diferencias en la deserción de los estudiantes por sexo e institución de procedencia (público o privado) y que otros aspectos, como no ingresar a la carrera elegida y poco conocimiento de la carrera matriculada resultan posibles causas de este fenómeno (Abarca y Sánchez, 2005).

En este artículo se analiza en primer lugar la deserción y seguidamente la reprobación. Respecto al concepto de deserción, el Centro Interuniversitario de Desarrollo (CINDA) (2006) lo define como el proceso de abandono, voluntario o forzoso de la carrera en la que se matricula un estudiante, por la influencia positiva o negativa de circunstancias internas o externas a él. En el caso de la educación superior, en forma más operativa, se entiende como la cantidad de estudiantes que abandona el sistema entre uno y otro período académico (semestre o año). El CINDA (2006) señala que la deserción debe analizarse desde tres perspectivas:

a) una óptica interna referida a los procesos de enseñanza (calidad educativa, estructura y pertinencia curricular, formación docente, etc.);

b) una visión externa relacionada con procesos ajenos a lo propiamente educativo (características del estudiantado, situación socioeconómica del país, acceso a financiamiento, etc.);

c) la interacción entre ambos tipos de factores, en donde la deserción debe ser considerada como el producto de la interrelación de múltiples factores, que relacionan el sistema educativo y la estructura social y económica de la sociedad.

De igual modo, el CINDA (2006) señala que la deserción, entendida como una forma de abandono de los estudios superiores, se caracteriza por una suspensión voluntaria y definitiva de los estudios y del sistema de educación superior por parte del alumno, salida de alumnos debido a deficiencias académicas y bajo rendimiento escolar, cambio de carrera (el alumno continúa en la misma institución, pero se incorpora a otra cohorte generacional) o de institución.

Por su parte, Grosset (1991), citado por Viale (2015), señala que se debe mirar la deserción como un factor de eficiencia institucional y, como tal, las estrategias para afrontarlas deberían contemplar tanto los pro- cesos educativos como las características de los estudiantes que propician el abandono, pues está más en función de lo que ocurre después de entrar a la universidad que la que le procede.

En esa misma línea de pensamiento, Canales y De los Ríos (2018), apuntan que la deserción no es unicausal, sino que responde a una multiplicidad de factores, por ejemplo, personales, culturales, sociales, económicos (tanto de los alumnos como de sus familias), así como los académicos e institucionales. Otras variables asociadas, según Canales y De los Ríos (2018) son los ambientes educativos, la edad, la adaptación social, la apatía de los programas curriculares, el cambio brusco del modelo pedagógico, los tipos de evaluación, los cursos que producen poco interés para su ejercicio profesional, entre otras (Páramo y Correa, 1999). Asimismo, González, Uribe y González (2005) establecen como otras causas de la deserción la formación académica previa del estudiante, los exámenes de ingreso, el nivel de aprendizaje adquirido, la excesiva orientación teórica, la escasa vinculación de los estudios con el mercado laboral y la falta de apoyo y orientación recibida por los profesores, entre otras.

Siempre en esa misma línea de ideas, González, Uribe y González (2005) definen la reprobación como la acción de cursar reiterativamente una actividad docente, sea por mal rendimiento del estudiante o por causas ajenas al ámbito académico. En la educación superior, puede estar referida a todas las actividades académicas de un período determinado (año, semestre o trimestre), o bien, a cada asignatura (Centro Interuniversitario de Desarrollo (CINDA), 2006).

Salazar y Espinoza (2017) por su parte, consideran que la reprobación y deserción escolar son fenómenos psicosociales complejos, en los que intervienen factores estructurales, sociales, familiares e individuales y que tienen repercusión en diferentes ámbitos de la realidad, tanto a nivel personal como institucional. Según ellos, es incorrecto atribuir estos fenómenos solo a situaciones personales -como la falta de dedicación, malos hábitos de estudio o compromiso con la institución por parte de los estudiantes- y es más conveniente buscar las soluciones en el nivel estudiantil. 
De acuerdo con Lentini, Martínez, Lentini, Tarifa y Crespo (2003), quienes consultaron a estudiantes y docentes, existen diversos factores que pueden influir en la repitencia de los alumnos, entre ellos están la falta de tiempo para dedicarse al curso debido a que deben atender otras asignaturas, poca dedicación personal, falta de atención y organización, falta de conocimientos previos, no entender los nuevos conceptos, no asistir a clases ni a consultas, poco acceso al docente, problemas familiares, de trabajo y económicos, falta ayuda por parte del profesor, pocas horas asignadas a las clases teóricas y metodología con que se imparten las lecciones.

En una línea similar, Talavera, Noreña, Plazola (2006), citados por Corral y Díaz (2009), determinaron algunos aspectos relacionados con la reprobación, tales como la organización del tiempo del estudiantado e inadecuados o ausencia de hábitos y técnicas de estudio de este, así como de estrategias didácticas inadecuadas por parte de los docentes. Corral y Díaz (2009), refiriéndose al estudio de Ruiz, Romano y Valenzuela (2006), indican que los motivos más importantes que ocasionan la reprobación universitaria son el desconocimiento del plan de estudios, las actitudes y el poco o nulo apoyo en métodos de estudio de los estudiantes y la falta de orientación vocacional que se da a nivel de secundaria.

En términos generales, de acuerdo con el CINDA (2006) entre los factores que inciden en la reprobación y la deserción, que se encuentran en la literatura, se pueden señalar los siguientes:

a) Personales. Las características personales del estudiante, por ejemplo, la falta de actitud, incompatibilidad del tiempo dedicado al trabajo y a los estudios, poco interés, poca acogida que le brinda la universidad, expectativas del estudiante respecto a la importancia de carrera que estudia, entre otras.

b) Institucionales. Entre las institucionales se destaca la deficiente orientación vocacional recibida antes de ingresar a la carrera que provoca, en algunos casos, que los alumnos se inscriban en las carreras sin sustentar su decisión en una adecuada información sobre las mismas, falta de preocupación institucional frente a resultados negativos, forma en que se organiza el trabajo académico, la pertinencia y la actualización del currículo, los apoyos materiales y administrativos, el cambio de carrera o de institución.

c) Pedagógicas. Las pedagógicas, por su parte, incluyen las características académicas previas del estudiante, la carencia de una práctica temprana y la ausencia de asignaturas que aproximan al ejercicio profesional desde los primeros años, los contenidos y la forma de estudiar.

d) Socioeconómicas y laborales. Entre ellas destacan las condiciones económicas desfavorables del estudiante, carencia de financiamiento, bajas expectativas de encontrar trabajo estable y con una remuneración adecuada, desarticulación familiar.

En cuanto al tema de las causas o factores de deserción, específicamente en cursos de Matemática, la cantidad de estudios ha ido en incremento en Costa Rica (Chaves, Castillo y Gamboa, 2008; PascuaCantero, 2016; Zamora-Araya y Villalobos-Madrigal, 2018). Existe una extensa literatura en descripciones o análisis de deserción universitaria en general, pero específicamente sobre sus causas, no se pueden pensar razones particulares.

Dadas las situaciones anteriormente expresadas y con el propósito de generar datos confiables sobre la reprobación y deserción, sus causas y producir recomendaciones, es que se hace necesario estudiar estos fenómenos en el curso de Matemática General, ofertado por la Escuela de Matemática de la Universidad Nacional de Costa Rica (UNA).

Con base en datos del departamento de Registro de la Universidad Nacional (2019), el curso de Matemática General es de los primeros al que se enfrentan los estudiantes al Ilegar a la UNA y es el que presenta los mayores índices de deserción en dicha área, lo que acorta el avance académico de los estudiantes (por ser requisito para otros cursos), genera una alta demanda y una inversión económica adicional para la universidad. Además, posee un alto porcentaje de reprobación, lo cual también produce complicaciones, tanto a nivel individual como social y emocional, en términos de las expectativas de los estudiantes y sus familias. 
Aunque la Escuela de Matemática de la UNA ha hecho un esfuerzo por sistematizar algunos datos y elaborar indicadores, este ha sido insuficiente. Por tanto, existe la necesidad de profundizar en este fenómeno para conocer su alcance real y, a partir de los resultados, producir acciones orientadas a atender esta problemática.

\section{METOdOLOGÍA}

Con base en la información del departamento de Registro Universidad Nacional (2019), el curso de Matemática General es el primero del área en la UNA; se imparte en los dos ciclos lectivos o semestres que la universidad tiene, y es requisito para otros cursos de carreras relacionadas con ingenierías, administración y enseñanza de las ciencias, entre otras. Los datos que se muestran en este artículo forman parte de los obtenidos en un proyecto de investigación, cuyo objetivo general fue identificar factores asociados a la deserción y repitencia en el curso de Matemática General, impartido por la Escuela de Matemática de la Universidad Nacional, con el propósito de proponer estrategias para la atención de esta problemática.

Particularmente, en esta investigación se pretendió analizar las razones por las cuales los estudiantes repiten y desertan en el curso de Matemática General en el periodo 2017. Para tales efectos, este artículo describe esta problemática, desde el enfoque del estudiante, para generar líneas generales en cuanto a las posibles causas de esta.

Para efectos de esta investigación, se trabajó con toda la población de estudiantes matriculados en el I y II ciclo lectivo del año 2017. Además, para el análisis de los datos se tomaron las siguientes consideraciones para la condición final:

a) Aprobado: estudiante que en la casilla correspondiente a la calificación del curso posee reportado una nota mayor o igual a 7.0.

b) Reprobado: estudiante que realizó todas las evaluaciones parciales del curso y que en la casilla correspondiente a la calificación del curso posee reportado una nota menor o igual a 6.75. Por el sistema de calificación y redondeo de la Univer- sidad Nacional , una nota superior a 6.75 se redondea a 7.0

c) Desertor: estudiante que no realizó una prueba parcial del curso y tampoco las posteriores a ella.

d) Retiro justificado: estudiante que en la casilla correspondiente a la calificación del curso poseen reportado un RJ (retiro justificado).

Adicionalmente, se consideraron las siguientes variables para el análisis de la condición final:

a) Género del estudiante: hombre, mujer.

b) Grupos de edad: de 16 a 20 años, 21 a 25 años y más de 25 años.

c) Área de procedencia: área de procedencia del estudiante según las siguientes categorías: Gran Área Metropolitana (GAM); fuera del Gran Área Metropolitana (NGAM).

d) Tipo de colegio de procedencia: público, privado, subvencionado.

e) Estrato del estudiante: I, II, III. La UNA clasifica a los estudiantes según los siguientes estratos

i. Estrato I: estudiantes de colegios privados, semipúblicos, científicos, humanísticos y extranjeros.

ii. Estrato II: estudiantes de colegios públicos.

iii. Estrato III: estudiantes de colegios nocturnos o que provienen de otras modalidades, como bachilleratos por madurez, Centros Integrados de Educación de Adultos (CINDEA)-Centros de Investigación para el Perfeccionamiento de la Educación Técnica (CIPET), telesecundarias, bachillerato a distancia, plan de estudios para la educación de jóvenes y adultos, entre otros.

f) Horario del curso: mañana, tarde, noche.

g) Grupo de carreras: se refiere a la carrera en la que está matriculado el estudiante, a saber, Administración, Biología, Cartografía y Diseño, Ciencias Geográficas, Comercio y Negocios Internacionales, Economía, Enseñanza de las 
Ciencias, Ingeniería Agronómica, Ingeniería Ciencias Forestales, Ingeniería Gestión Ambiental, Ingeniería Topografía y Catastro.

h) Beca: goza o no de este tipo de beneficio, ya sea de forma total o parcial.

i) Situación laboral: aparte de estudiar, trabaja de forma remunerada y permanente, más de 36 horas a la semana, o no.

j) Año de carrera: I, II, III o IV.

k) Percepción personal como estudiante: Muy buen estudiante, buen estudiante, estudiante regular, mal estudiante y pésimo estudiante.

I) Percepción familiar del estudiante: Muy buen estudiante, buen estudiante, estudiante regular, mal estudiante y pésimo estudiante.

m) Rendimiento académico en secundaria: muy bueno, bueno, regular, malo y muy malo.

n) Estudiaba diariamente la materia del curso: muy de acuerdo, de acuerdo, ni de acuerdo ni en desacuerdo, en desacuerdo y muy en desacuerdo.

o) Tiempo semanal de estudio: menos de 2 horas, de 2 a 5 horas, de 5 a menos de 10 horas y 10 o más horas.

p) Asistencia a clases: muy buena, buena, regular, mala y muy mala.

Durante el I ciclo de 2017, se matricularon en el curso Matemática General 1251 estudiantes; de ellos desertaron 572 y 305 lo habían reprobado en el ciclo anterior. Para el II ciclo de ese mismo año, la matrícula fue de 731 estudiantes, de los cuales 306 desertaron y 137 reprobaron.

Antes de iniciar la construcción de los cuestionarios se realizó una revisión de la literatura relacionada con la temática, con el propósito de identificar instrumentos que ya hubiesen sido aplicados y que sirvieran base, según los ítems incluidos, para la construcción de los que se utilizaron en la investigación. Los cuestionarios, en forma general, estaban compuestos por preguntas de información personal y académica del estudiante e ítems relacionados con su trayectoria en la universidad, específicamente en el curso de Matemática General, que incluían, entre otros aspectos, posibles causas de deserción y repitencia, para que el estudiante valorara el grado de influencia de estos.

Una vez diseñados los instrumentos en su primera versión y después de delimitar la información, formular las preguntas, determinar el número y su orden, se procedió a realizar una validación con expertos, la cual consistió en evaluar la redacción, pertinencia y coherencia de los ítems.

Posteriormente, se realizó una prueba piloto. Esta prueba se realizó a siete grupos del curso de "Matemática para Informática", para un total de 131 estudiantes. Esta población tenía características muy similares a los estudiantes de Matemática General, a los cuales va dirigido los cuestionarios definitivos.

Esta prueba tenía como propósito identificar, entre otros elementos, el tipo de pregunta más adecuado, si los enunciados eran correctos y comprensibles, revisar la extensión de las preguntas, si son correctas las categorías asignadas a ciertos ítems y si la duración en el tiempo de respuesta de los cuestionarios es aceptable.

Después de realizada la prueba piloto, se digitó la base de datos para realizar un análisis descriptivo y determinar incoherencias en los resultados; además, durante la realización del cuestionario se determinaron algunas preguntas que no estaban correctamente redactadas.

A los estudiantes desertores y reprobados se les aplicó un cuestionario vía correo electrónico en el cual se les consultó sobre aspectos generales y posibles causas de deserción y reprobación. Al ser la muestra autoseleccionada y vía web, el estudiante podía decidir llenar el cuestionario por su propia motivación $\mathrm{o}$ incluso no finalizarlo.

Además, se garantizó que la información suministrada sería manejada confidencialmente, sin evidenciar casos particulares y únicamente para los propósitos de la investigación. La muestra resultante es autoseleccionada, ya que el cuestionario lo responden los educandos que así lo deseen, por lo que una limitante es la no inferencia de los resultados, sin embargo, algunos estudios han demostrado (Chang y Krosnick, 
2009; Shropshire et al., 2009, citados por De Marchis, 2012) que los individuos que llevan a cabo una encuesta autoseleccionada por internet o mediante correo electrónico tienen más conocimientos, compromiso o afinidad con el tema de la investigación que las personas que habían sido elegidas de forma aleatoria. Por lo tanto, es de esperar que un estudiante que tiene más interés y compromiso con el tema de deserción y reprobación tenga más probabilidades de iniciar y terminar el cuestionario enviado vía correo electrónico.

Entre los aspectos que incluyeron para la consulta a los estudiantes se circunscriben información general (sexo, edad, área de procedencia, entre otros) así como posibles causas de la deserción y reprobación según la literatura consultada.

Para el análisis de los datos se utilizó el software libre PSPP para generar distribuciones de frecuencias y tablas de contingencia. A continuación, se presenta una selección de los datos obtenidos.

\section{RESULTADOS Y DISCUSIÓN}

\section{CARACTERIZACIÓN DE LA POBLACIÓN}

El análisis comparó las tendencias entre el I ciclo y el II ciclo del año 2017 en el curso de Matemática General. Si bien es cierto no constituye un estudio longitudinal, se ha constó, como parte del estudio, que la mayoría de los estudiantes que desertan o reprueban el curso en el I ciclo lo vuelven a matricular en el ciclo siguiente.

Primeramente, se realizará una descripción de los grupos de estudiantes del I ciclo y II ciclo del año 2017. Durante el I ciclo tenemos 647 estudiantes, donde aproximadamente el $46,0 \%$ desertó y el $24,0 \%$ reprobó. Durante el II ciclo se mantiene una proporción elevada de desertores $(0,42)$, la reprobación baja a 18,7\%, pero es interesante observar que la condición de aprobado pasa de $26,1 \%$ en el I ciclo a $37,2 \%$ en el II ciclo, tendencias que se mantienen si se realiza la comparación por sexo, según se observa en el Tabla 1:

\section{Tabla 1. Distribución de estudiantes por sexo según condición y ciclo lectivo, Matemática General, Universidad Nacional, 2017}

\begin{tabular}{|c|c|c|c|c|c|c|c|}
\hline \multirow{2}{*}{ Ciclo lectivo } & \multirow{2}{*}{ Condición } & \multicolumn{2}{|c|}{ Hombre } & \multicolumn{2}{|c|}{ Mujer } & \multicolumn{2}{|c|}{ Total } \\
\hline & & Absoluto & Porcentaje & Absoluto & Porcentaje & Absoluto & Porcentaje \\
\hline \multirow{5}{*}{ I } & Aprobado & 145 & 22,4 & 181 & 30,0 & 326 & 26,1 \\
\hline & Reprobado & 136 & 21,0 & 169 & 28,0 & 305 & 24,4 \\
\hline & Desertor & 342 & 52,9 & 230 & 38,1 & 572 & 45,7 \\
\hline & Retiro justificado & 24 & 3,7 & 24 & 4,0 & 48 & 3,8 \\
\hline & Total & 647 & 100,0 & 604 & 100,0 & 1251 & 100,0 \\
\hline \multirow{5}{*}{ II } & Aprobado & 118 & 29,8 & 154 & 46,0 & 272 & 37,2 \\
\hline & Reprobado & 72 & 18,2 & 65 & 19,4 & 137 & 18,7 \\
\hline & Desertor & 198 & 50,0 & 108 & 32,2 & 306 & 41,9 \\
\hline & Retiro justificado & 8 & 2,0 & 8 & 2,4 & 16 & 2,2 \\
\hline & Total & 396 & 100,0 & 335 & 100,0 & 731 & 100,0 \\
\hline
\end{tabular}

Fuente: Elaboración propia

Al realizar la distribución por grupos de edad, se percibe que la población que matricula el curso de Matemática General es una población relativamente joven donde la mayoría tiene menos de 25 años.
Destaca que casi el 58,0\% del grupo de 21 años a 25 años deserta; también se puede notar que el grupo de 16 años a 20 años es el que tiene el mayor porcentaje de aprobación, un 30,9\%. 
Tabla 2. Distribución de estudiantes por edad según condición y ciclo lectivo, Matemática General, Universidad Nacional, 2017

\begin{tabular}{|c|c|c|c|c|c|c|c|c|c|c|c|}
\hline \multirow{2}{*}{ Ciclo lectivo } & \multirow{2}{*}{ Condición } & \multicolumn{2}{|c|}{$\begin{array}{l}\text { De } 16 \text { años a } \\
20 \text { años }\end{array}$} & \multicolumn{2}{|c|}{$\begin{array}{c}\text { De } 21 \text { años a } \\
25 \text { años }\end{array}$} & \multicolumn{2}{|c|}{$\begin{array}{l}\text { Más de } 25 \\
\text { años }\end{array}$} & \multicolumn{2}{|c|}{ No respondió } & \multicolumn{2}{|c|}{ Total } \\
\hline & & Abs. & Por. & Abs. & Por. & Abs. & Por. & Abs. & Por. & Abs. & Por. \\
\hline \multirow{5}{*}{ I } & Aprobado & 253 & 30,9 & 43 & 21,5 & 11 & 19,0 & 19 & 10,9 & 326 & 26,1 \\
\hline & Reprobado & 235 & 28,7 & 39 & 19,5 & 16 & 27,6 & 15 & 8,6 & 305 & 24,4 \\
\hline & Desertor & 326 & 39,8 & 115 & 57,5 & 29 & 50,0 & 102 & 58,6 & 572 & 45,7 \\
\hline & Retiro & 5 & 0,6 & 3 & 1,5 & 2 & 3,4 & 38 & 21,8 & 48 & 3,8 \\
\hline & Total & 819 & 100,0 & 200 & 100,0 & 58 & 100,0 & 174 & 100,0 & 1251 & 100,0 \\
\hline \multirow{5}{*}{ II } & Aprobado & 215 & 44,2 & 34 & 27,4 & 10 & 31,3 & 13 & 14,6 & 272 & 37,2 \\
\hline & Reprobado & 101 & 20,8 & 15 & 12,1 & 4 & 12,5 & 17 & 19,1 & 137 & 18,7 \\
\hline & Desertor & 166 & 34,2 & 74 & 59,7 & 18 & 56,3 & 48 & 53,9 & 306 & 41,9 \\
\hline & Retiro & 4 & 0,8 & 1 & 0,8 & 0 & 0,0 & 11 & 12,4 & 16 & 2,2 \\
\hline & Total & 486 & 100,0 & 124 & 100,0 & 32 & 100,0 & 89 & 100,0 & 731 & 100,0 \\
\hline
\end{tabular}

Fuente: Elaboración propia

En cuanto a la zona de procedencia, se encuentran grandes diferencias entre los desertores y reprobados de las dos regiones (GAM y NGAM). Se puede observar que en el II ciclo el porcentaje de aprobado aumenta en las dos áreas, con una mayoría de estudiantes provenientes de fuera de la GAM, lo que po- dría ser un indicador que el estudiante, en el II Ciclo, ya ha alcanzado un grado de madurez y adaptación al sistema universitario que le permite la aprobación en el curso. Sin embargo, es un aspecto en el que se debe profundizar.

Tabla 3. Distribución de estudiantes por región según condición y ciclo lectivo, Matemática General, Universidad Nacional, 2017

\begin{tabular}{|c|c|c|c|c|c|c|c|c|c|}
\hline \multirow{2}{*}{ Ciclo lectivo } & \multirow{2}{*}{ Condición } & \multicolumn{2}{|c|}{ GAM } & \multicolumn{2}{|c|}{ NGAM } & \multicolumn{2}{|c|}{ No respondió } & \multicolumn{2}{|c|}{ Total } \\
\hline & & Abs. & Por. & Abs. & Por. & Abs. & Por. & Abs. & Por. \\
\hline \multirow{5}{*}{ I } & Aprobado & 211 & 29,8 & 96 & 26,2 & 19 & 10,9 & 326 & 26,1 \\
\hline & Reprobado & 182 & 25,7 & 107 & 29,2 & 16 & 9,1 & 305 & 24,4 \\
\hline & Desertor & 308 & 43,4 & 162 & 44,1 & 102 & 58,3 & 572 & 45,7 \\
\hline & Retiro & 8 & 1,1 & 2 & 0,5 & 38 & 21,7 & 48 & 3,8 \\
\hline & Total & 709 & 100,0 & 367 & 100,0 & 175 & 100,0 & 1251 & 100,0 \\
\hline \multirow{5}{*}{ II } & Aprobado & 156 & 39,6 & 102 & 42,0 & 14 & 14,9 & 272 & 37,2 \\
\hline & Reprobado & 68 & 17,3 & 51 & 21,0 & 18 & 19,1 & 137 & 18,7 \\
\hline & Desertor & 168 & 42,6 & 87 & 35,8 & 51 & 54,3 & 306 & 41,9 \\
\hline & Retiro & 2 & 0,5 & 3 & 1,2 & 11 & 11,7 & 16 & 2,2 \\
\hline & Total & 394 & 100,0 & 243 & 100,0 & 94 & 100,0 & 731 & 100,0 \\
\hline
\end{tabular}

Fuente: Elaboración propia

Un aspecto significativo a la hora de considerar la reprobación y deserción de los estudiantes es el tiempo que ha transcurrido desde su egreso de secundaria, pues los estudiantes que tienen más de 8 años de haber concluido esa etapa, tienen un porcentaje de deserción de casi un 62,0\%. 
En relación con el tipo de colegio, el menor porcentaje de deserción se presenta en los estudiantes provenientes de colegios subvencionados; igualmente, la menor reprobación y la mayor aprobación se da en este grupo de estudiantes. Las condiciones más desfavorables se presentan en los colegios públicos, es decir, un porcentaje alto de deserción y reprobación y porcentajes bajos de aprobación.

Tabla 4. Distribución de estudiantes por tipo de colegio según condición y ciclo lectivo, Matemática General, Universidad Nacional, 2017

\begin{tabular}{|c|c|c|c|c|c|c|c|c|c|c|c|}
\hline \multirow{2}{*}{ Ciclo lectivo } & \multirow{2}{*}{ Condición } & \multicolumn{2}{|c|}{ Público } & \multicolumn{2}{|c|}{ Privado } & \multicolumn{2}{|c|}{ Subvencionado } & \multicolumn{2}{|c|}{ No respondió } & \multicolumn{2}{|c|}{ Total } \\
\hline & & Abs. & Por. & Abs. & Por. & Abs. & Por. & Abs. & Por. & Abs. & Por. \\
\hline \multirow{5}{*}{ I } & Aprobado & 210 & 25,4 & 41 & 28,9 & 53 & 55,8 & 22 & 11,7 & 326 & 26,1 \\
\hline & Reprobado & 237 & 28,7 & 36 & 25,4 & 14 & 14,7 & 18 & 9,6 & 305 & 24,4 \\
\hline & Desertor & 370 & 44,8 & 64 & 45,1 & 28 & 29,5 & 110 & 58,5 & 572 & 45,7 \\
\hline & Retiro & 9 & 1,1 & 1 & 0,7 & 0 & 0,0 & 38 & 20,2 & 48 & 3,8 \\
\hline & Total & 826 & 100,0 & 142 & 100,0 & 95 & 100,0 & 188 & 100,0 & 1251 & 100,0 \\
\hline \multirow{5}{*}{ ॥ } & Aprobado & 208 & 39,5 & 31 & 41,9 & 14 & 45,2 & 19 & 19,2 & 272 & 37,2 \\
\hline & Reprobado & 106 & 20,1 & 11 & 14,9 & 2 & 6,5 & 18 & 18,2 & 137 & 18,7 \\
\hline & Desertor & 209 & 39,7 & 31 & 41,9 & 15 & 48,4 & 51 & 51,5 & 306 & 41,9 \\
\hline & Retiro & 4 & 0,8 & 1 & 1,4 & 0 & 0,0 & 11 & 11,1 & 16 & 2,2 \\
\hline & Total & 527 & 100,0 & 74 & 100,0 & 31 & 100,0 & 99 & 100,0 & 731 & 100,0 \\
\hline
\end{tabular}

Fuente: Elaboración propia

En el Tabla 5 se muestra la distribución por carrera, y se observa que no hay un comportamiento uniforme al pasar de un ciclo a otro. Sin embargo, se destaca el caso de algunas carreras como Administración, la cual se encuentra entre las primeras en los niveles de deserción, al igual que Ingeniería Agronómica. En la mayoría de las carreras se observa una disminución en los porcentajes de reprobación en el II ciclo.

Tabla 5. Distribución de estudiantes por condición según carrera, en el curso de Matemática General, Universidad Nacional, 2017

\begin{tabular}{lcccc}
\hline \multirow{2}{*}{ Carrera } & \multicolumn{2}{c}{ I Ciclo } & \multicolumn{2}{c}{ II Ciclo } \\
\cline { 2 - 5 } & Desertor & Reprobado & Desertor & Reprobado \\
\hline Administración & 52,0 & 21,6 & 45,5 & 17,0 \\
\hline Biología & 37,0 & 32,0 & 43,1 & 13,8 \\
\hline Cartografía y diseño & 39,2 & 31,4 & 25,7 & 40,0 \\
\hline Ciencias geográficas & 41,7 & 30,0 & 35,3 & 32,4 \\
\hline Comercio y negocios internacionales & 39,2 & 27,5 & 47,0 & 12,1 \\
\hline Economía & 35,0 & 20,6 & 35,9 & 23,4 \\
\hline Enseñanza de las ciencias & 38,3 & 26,7 & 41,2 & 23,5 \\
\hline Ingeniería agronómica & 53,2 & 28,6 & 40,0 & 16,0 \\
\hline Ingeniería en ciencias forestales & 49,0 & 25,5 & 48,5 & 12,1 \\
\hline Ingeniería en gestión ambiental & 31,0 & 38,1 & 24,0 & 20,0 \\
\hline Ingeniería en topografía y catastro & 53,2 & 25,5 & 41,1 & 16,1 \\
\hline
\end{tabular}

Fuente: Elaboración propia 
Los estudiantes que poseen beca tienen porcentajes de deserción un poco menores a los estudiantes no becados, sin embargo, los porcentajes de aprobación son muy similares.

La situación laboral es otra variable que debería estar fuertemente ligada a los niveles de deserción. En este caso, la minoría es la que trabaja; a pesar de esto tienen una tasa de deserción del 64,3 por cada 100 y una tasa de aprobación del 16,8 por cada 100; se observa que de todos los estudiantes que terminan el curso el 50\% aprueba y el otro 50\% reprueba.

Tabla 6. Distribución de estudiantes por condición laboral según condición y ciclo lectivo, Matemática General, Universidad Nacional, 2017

\begin{tabular}{|c|c|c|c|c|c|c|c|c|c|}
\hline \multirow{2}{*}{ Ciclo lectivo } & \multirow{2}{*}{ Condición } & \multicolumn{2}{|c|}{ Trabaja } & \multicolumn{2}{|c|}{ No trabaja } & \multicolumn{2}{|c|}{ No respondió } & \multicolumn{2}{|c|}{ Total } \\
\hline & & Abs. & Por. & Abs. & Por. & Abs. & Por. & Abs. & Por. \\
\hline & Aprobado & 31 & 16,8 & 271 & 31,2 & 24 & 12,1 & 326 & 26,1 \\
\hline & Reprobado & 32 & 17,3 & 251 & 28,9 & 22 & 11,1 & 305 & 24,4 \\
\hline & Desertor & 119 & 64,3 & 339 & 39,1 & 114 & 57,6 & 572 & 45,7 \\
\hline & Retiro & 3 & 1,6 & 7 & 0,8 & 38 & 19,2 & 48 & 3,8 \\
\hline & Total & 185 & 100,0 & 868 & 100,0 & 198 & 100,0 & 1251 & 100,0 \\
\hline \multirow{5}{*}{$\pi$} & Aprobado & 31 & 29,0 & 219 & 42,5 & 22 & 20,2 & 272 & 37,2 \\
\hline & Reprobado & 10 & 9,3 & 107 & 20,8 & 20 & 18,3 & 137 & 18,7 \\
\hline & Desertor & 66 & 61,7 & 184 & 35,7 & 56 & 51,4 & 306 & 41,9 \\
\hline & Retiro & 0 & 0,0 & 5 & 1,0 & 11 & 10,1 & 16 & 2,2 \\
\hline & Total & 107 & 100,0 & 515 & 100,0 & 109 & 100,0 & 731 & 100,0 \\
\hline
\end{tabular}

Fuente: Elaboración propia

Como ya se mencionó, para el proceso de admisión estudiantil, la UNA clasifica a los estudiantes en tres estratos. Al analizar el estrato del cual proviene el estudiante, en la Tabla 7 se observa que los menores niveles de reprobación se dan en el Estrato I, para ambos ciclos. Para los del Estrato II, es interesante no- tar cómo para el II ciclo estos estudiantes logran tener un porcentaje de aprobación muy similar a los del I estrato. Además, se puede notar que en el caso de la deserción, en ambos ciclos los estudiantes del Estrato III, tienen los mayores niveles de deserción con porcentajes mayores al 48,0\%.

Tabla 7. Distribución de estudiantes por estrato según condición y ciclo lectivo, Matemática General, Universidad Nacional, 2017

\begin{tabular}{|c|c|c|c|c|c|c|c|c|c|c|c|}
\hline \multirow{2}{*}{ Ciclo lectivo } & \multirow{2}{*}{ Condición } & \multicolumn{2}{|c|}{ Estrato 1} & \multicolumn{2}{|c|}{ Estrato 2} & \multicolumn{2}{|c|}{ Estrato 3} & \multicolumn{2}{|c|}{ No respondió } & \multicolumn{2}{|c|}{ Total } \\
\hline & & Abs. & Por. & Abs. & Por. & Abs. & Por. & Abs. & Por. & Abs. & Por. \\
\hline \multirow{5}{*}{ I } & Aprobado & 90 & 40,7 & 165 & 25,1 & 14 & 11,2 & 57 & 23,1 & 326 & 26,1 \\
\hline & Reprobado & 49 & 22,2 & 184 & 28,0 & 30 & 24,0 & 42 & 17,0 & 305 & 24,4 \\
\hline & Desertor & 71 & 32,1 & 288 & 43,8 & 75 & 60,0 & 138 & 55,9 & 572 & 45,7 \\
\hline & Retiro & 11 & 5,0 & 21 & 3,2 & 6 & 4,8 & 10 & 4,0 & 48 & 3,8 \\
\hline & Total & 221 & 100,0 & 658 & 100,0 & 125 & 100,0 & 247 & 100,0 & 1251 & 100,0 \\
\hline \multirow{5}{*}{ II } & Aprobado & 33 & 45,2 & 148 & 43,8 & 19 & 27,9 & 72 & 28,6 & 272 & 37,2 \\
\hline & Reprobado & 11 & 15,1 & 76 & 22,5 & 15 & 22,1 & 35 & 13,9 & 137 & 18,7 \\
\hline & Desertor & 28 & 38,4 & 112 & 33,1 & 33 & 48,5 & 133 & 52,8 & 306 & 41,9 \\
\hline & Retiro & 1 & 1,4 & 2 & 0,6 & 1 & 1,5 & 12 & 4,8 & 16 & 2,2 \\
\hline & Total & 73 & 100,0 & 338 & 100,0 & 68 & 100,0 & 252 & 100,0 & 731 & 100,0 \\
\hline
\end{tabular}

Fuente: Elaboración propia 
Una vez realizada la descripción general de la población de estudiantes del curso Matemática General, se analiza el grupo de estudiantes que estuvieron interesados en contestar el cuestionario, lo que permite formular hipótesis de las causas de deserción y reprobación desde el punto de vista del estudiantado.

\section{PERCEPCIÓN DE LOS ESTUDIANTES SOBRE LAS CAUSAS DE DESERCIÓN Y REPROBACIÓN}

Para el desarrollo de este apartado se consideraron las respuestas de 465 cuestionarios respondidos. Es importante recalcar que estas muestras son autoseleccionadas, es decir, el cuestionario se envió por correo electrónico a todos los estudiantes y fue contestado por aquellos estudiantes interesados en responder. Esta limitación de no corresponder a muestras aleatorias de la población limita la inferencia de los resultados, al no poder realizar inferencia para toda la población. Sin embargo, la información recolectada es igualmente importante y da un panorama de lo que podría ser la perspectiva del estudiantado sobre estos temas de deserción y reprobación.

Es importante para iniciar el análisis indicar que más del $70,0 \%$ de los estudiantes desertores y reprobados estaban matriculados en la carrera que ellos seleccionaron como primera opción de estudio en la UNA.

En la Figura 1, y con base en la muestra consultada, se observa que el menor porcentaje de estudiantes reprobados y desertores estuvieron matriculados en horario nocturno. En el caso de la reprobación el mayor porcentaje de estudiantes estaba matriculado en el horario de la mañana, mientras que el mayor porcentaje de desertores, estaban matriculados en cursos de la tarde.

\section{Figura 1. Distribución de estudiantes por condición y ciclo según horario de curso Matemática General, Universidad Nacional, 2017}

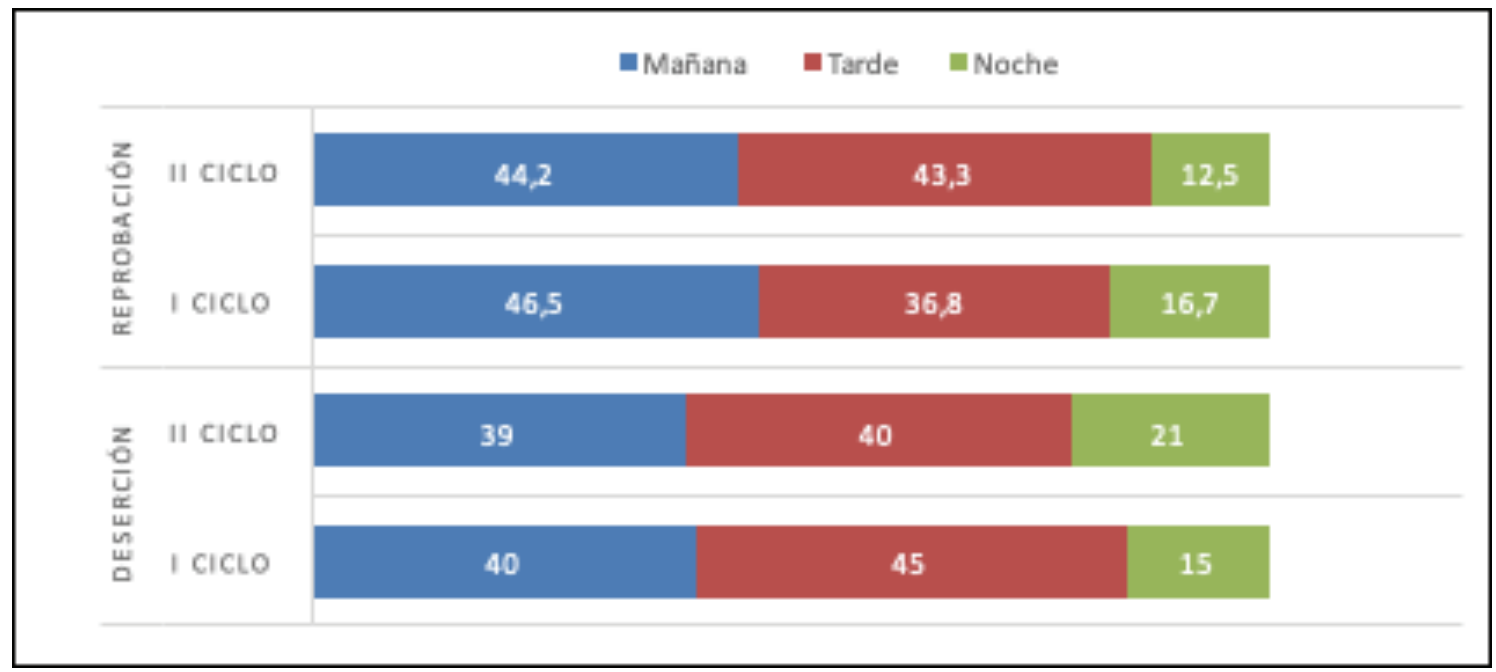

Fuente: Elaboración propia

Como ya se ha mencionado, Matemática General es el primer curso que llevan los estudiantes en el área cuando ingresan a la UNA y que es cursado, por la mayoría de los estudiantes, en los primeros ciclos de cada año. En la Tabla 8, se puede notar que los estudiantes desertores son, en mayoría, del primer año de carrera, con un $76,3 \%$ y $82,1 \%$ en el I y II ciclo, respectivamente. Lo que refleja que la problemática se presenta, en mayor porcentaje, para los estudian- tes que ingresar por primera vez a la universidad, por lo que se debe prestar especial atención a esta población.

En cuanto a la reprobación podemos notar que en el I ciclo aproximadamente el 65,0\% de los estudiantes que reprobaron el curso estaban en el segundo, tercer y cuarto años de carrera. Para el II ciclo, los estudiantes que reprobaron en su mayoría eran de primer año 83,3\%. 
Tabla 8. Distribución de estudiantes del curso Matemática General por año de carrera, según condición y ciclo, Universidad Nacional, 2017

\begin{tabular}{lcccc}
\hline \multirow{2}{*}{ Año de carrera } & \multicolumn{2}{c}{ Deserción } & \multicolumn{2}{c}{ Reprobación } \\
\cline { 2 - 5 } & I ciclo & II ciclo & I Ciclo & II ciclo \\
\hline Desertó de la universidad & 2,5 & 1,5 & - & - \\
\hline I año & 76,3 & 82,1 & 36,0 & 83,3 \\
\hline II año & 14,7 & 10,4 & 52,6 & 14,3 \\
\hline III año & 4,3 & 1,5 & 8,8 & 1,6 \\
\hline IV año & 2,2 & 4,5 & 2,6 & 0,8 \\
\hline Total & 100,0 & 100,0 & 100,0 & 100,0 \\
\hline
\end{tabular}

Fuente: Elaboración propia

En cuanto a los ingresos familiares de los estudiantes desertores, así como estudiantes repitentes, la mayoría (en promedio $77 \%$ ) de ellas cuentan con ingresos mensuales menores a US\$1000 (equivalente a dos salarios mínimos de Costa Rica). En el cuestionario original se les hizo la consulta en colones, que es la moneda de este país. La presentación en dólares obedece a posibles intereses de referencia en otros países.

Para las familias de estudiantes desertores del I ciclo, el $77,4 \%$ contaba con esos ingresos mientras que para el II ciclo correspondía al 76,1\%. En el caso de estudiantes reprobados, la mayoría de las familias está en el mismo rango de ingresos, $68,7 \%$ y $75,0 \%$ respectivamente.
Para la muestra de estudio, el 50,0\% aproximadamente de los estudiantes desertores y reprobados manifiesta que no cuenta con el apoyo por parte de la familia, aunque este amparo no necesariamente es económico, pues se centra más en aspectos motivacionales o afectivos, entre otros.

Un aspecto adicional para considerar fue la percepción que tiene los estudiantes sobre sí mismos. En esta pregunta se utilizó una escala de Likert con cinco categorías: "muy buen estudiante", "buen estudiante", "estudiante regular", "mal estudiante" y "pésimo estudiante". En la Figura 2, se nota que la mayoría de los estudiantes encuestados se auto percibe como "bueno" o "muy buenos estudiantes", tanto los desertores como los reprobados, sin importar el ciclo lectivo.

Figura 2. Distribución de estudiantes del curso Matemática General, por autopercepción como estudiante según condición y ciclo, Universidad Nacional, 2017

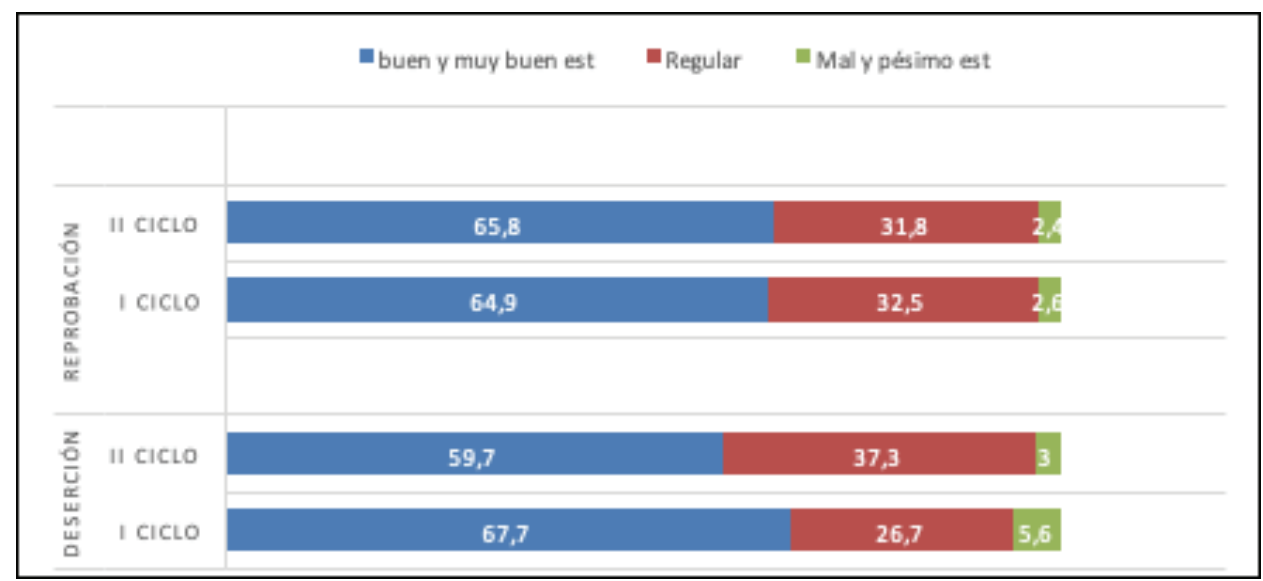

Fuente: Elaboración propia 
Tanto la percepción que tiene el estudiante como la familia, de su desempeño estudiantil, se ubica en mayor porcentaje en las categorías "muy buen estudiante" y "buen estudiante". La mayor parte de los estudiantes se auto-perciben como "muy buenos estudiantes" o "buenos estudiantes" (entre un 59,7\% y un $67,7 \%$ ), independientemente en la categoría de rendimiento del curso en la que se ubiquen (desertor o reprobado) o el semestre en que cursaron la asignatura (I o II ciclo). En el caso de la percepción que los estudiantes consideran que su familia tiene de ellos, la mayoría considera que su familia los asume como "muy buen estudiante" o "buen estudiante", pero en un porcentaje aún mayor al de su propia percepción (entre un $73,2 \%$ y un $82,2 \%$ ). En la Tabla 9 , se detalla esta información.

Tabla 9. Distribución de estudiantes del curso Matemática General, por percepción familiar del estudiante, según condición y ciclo, Universidad Nacional, 2017

\begin{tabular}{lcccc}
\hline \multirow{2}{*}{ Percepción familiar } & \multicolumn{2}{c}{ Deserción } & \multicolumn{2}{c}{ Repitencia } \\
\cline { 2 - 5 } & I Ciclo & II Ciclo & I Ciclo & II Ciclo \\
\hline Muy buen estudiante & 31,7 & 23,9 & 26,3 & 31,7 \\
\hline Buen estudiante & 50,4 & 49,3 & 52,6 & 45,8 \\
\hline Estudiante regular & 15,1 & 23,9 & 18,4 & 16,7 \\
\hline Mal estudiante & 2,5 & 1,5 & 1,8 & 4,2 \\
\hline Pésimo estudiante & 0,4 & 1,5 & 0,9 & 1,6 \\
\hline Total & 100,0 & 100,0 & 100 & 100 \\
\hline
\end{tabular}

Fuente: Elaboración propia

Matemática General se ha percibido como un curso de repaso para que los estudiantes puedan lograr un mismo nivel de conocimiento y asegurar que se aborden los contenidos mínimos para afrontar otros cursos de matemática y disciplinares de los respectivos planes de estudios de las carreras. Por ello, una variable por considerar es el rendimiento académico en secundaria, ya que se esperaría que a un mejor rendimiento en secundaria así sería el de este curso.
Desde la perspectiva de los estudiantes, el rendimiento académico en secundaria, tanto para los estudiantes desertores como para los repitentes en el I y II ciclo lectivo, lo consideran "muy bueno" o "bueno" en un 50,0\% aproximadamente, mientras que un $35,0 \%$, aproximadamente, indica que fue "regular". En la siguiente Tabla, se puede ver con detalle las respuestas respecto a este ítem.

Tabla 10. Distribución de estudiantes según rendimiento académico secundaria, según condición y ciclo, Matemática General, Universidad Nacional, 2017

\begin{tabular}{lcccc}
\hline \multirow{2}{*}{ Rendimiento académico } & \multicolumn{2}{c}{ Deserción } & \multicolumn{2}{c}{ Repitencia } \\
\cline { 2 - 5 } & I Ciclo & II Ciclo & I Ciclo & II Ciclo \\
\hline Muy bueno & 17,6 & 17,9 & 25,4 & 30,0 \\
\hline Bueno & 32,7 & 32,8 & 24,6 & 42,5 \\
\hline Regular & 34,5 & 29,9 & 37,8 & 20,8 \\
\hline Malo & 11,2 & 16,4 & 8,8 & 6,7 \\
\hline Muy malo & 4,0 & 3,0 & 3,4 & 0,0 \\
\hline Total & 100.0 & 100.0 & 100 & 100 \\
\hline
\end{tabular}

Fuente: Elaboración propia 
A pesar de que el rendimiento académico en secundaria se puede considerar aceptable, en promedio, el $85,0 \%$ de los estudiantes desertores y reprobados indican que los conocimientos de secundaria no fueron suficientes para aprobar el curso de Matemática General.
Ante la consulta de si estudiaban a diario el curso Matemática General, menos de una cuarta parte de los estudiantes expresó estar "de acuerdo" o "muy de acuerdo", en ambos ciclos, tanto desertores como reprobados. En la Tabla 11, se observan los porcentajes correspondientes.

Tabla 11. Distribución de estudiantes según estudiaban diariamente, por condición y ciclo, Matemática General, Universidad Nacional, 2017

\begin{tabular}{lcccc}
\hline \multirow{2}{*}{ Estudio diario } & \multicolumn{2}{c}{ Deserción } & \multicolumn{2}{c}{ Repitencia } \\
\cline { 2 - 5 } & I Ciclo & II Ciclo & I Ciclo & II Ciclo \\
\hline Muy de acuerdo & 2,9 & 4,5 & 7,9 & 3,3 \\
\hline De acuerdo & 19,1 & 20,9 & 14,9 & 19,2 \\
\hline Ni de acuerdo ni en desacuerdo & 45,0 & 37,3 & 42,1 & 43,3 \\
\hline En desacuerdo & 25,9 & 29,9 & 27,2 & 25,0 \\
\hline Muy en desacuerdo & 7,2 & 7,4 & 7,9 & 9,2 \\
\hline Total & 100,0 & 100,0 & 100,0 & 100,0 \\
\hline
\end{tabular}

Fuente: Elaboración propia

El curso de Matemática General contempla en el descriptor del curso un mínimo de 6 horas de estudio independiente por semana. Sin embargo, los es- tudiantes indican, en una gran mayoría, que estudian menos de 5 horas, lo cual se detalla en el Tabla 12:

Tabla 12. Distribución de estudiantes según tiempo semanal de estudio, por condición y ciclo, Matemática General, Universidad Nacional, 2017

\begin{tabular}{lcccc}
\hline \multirow{2}{*}{ Tiempo } & \multicolumn{2}{c}{ Deserción } & \multicolumn{2}{c}{ Repitencia } \\
\cline { 2 - 5 } & I Ciclo & II Ciclo & I Ciclo & II Ciclo \\
\hline Menos de 2 horas & 35,3 & 40,3 & 29,8 & 27,5 \\
\hline De 2 a menos de 5 horas & 50,4 & 46,3 & 53,5 & 53,3 \\
\hline De 5 a menos de 10 horas & 12,9 & 11,9 & 14,9 & 16,7 \\
\hline 10 o más horas & 1,4 & 1,5 & 1,8 & 2,5 \\
\hline Total & 100,0 & 100,0 & 100,0 & 100,0 \\
\hline
\end{tabular}

Fuente: Elaboración propia

Otro factor que podría estar relacionado con la deserción y la reprobación es la asistencia a clases. En la Figura 3, se muestra que los estudiantes desertores mostraron menores porcentajes en la categoría de asistencia a clases "muy buena" o "buena" (59,7\% $67,3 \%$ ) que los estudiantes que reprobaron el curso $(78,0 \%-85,0 \%)$, como es de esperar. No obstante, la percepción de los estudiantes desertores no parece ser muy objetiva, pues por su condición de aban- dono de curso, su asistencia no podría considerarse buena. Según la respuesta de los estudiantes existe una gran población que no lo hace con la regularidad que el curso requiere. Un aspecto por recalcar es que en este curso, la asistencia a clases no es obligatoria, pero por las temáticas tratadas en él se considera primordial hacerlo, lo cual es una responsabilidad directa del estudiante. 
Figura 3. Distribución de estudiantes según asistencia a clases, por condición y ciclo, Matemática General, Universidad Nacional, 2017

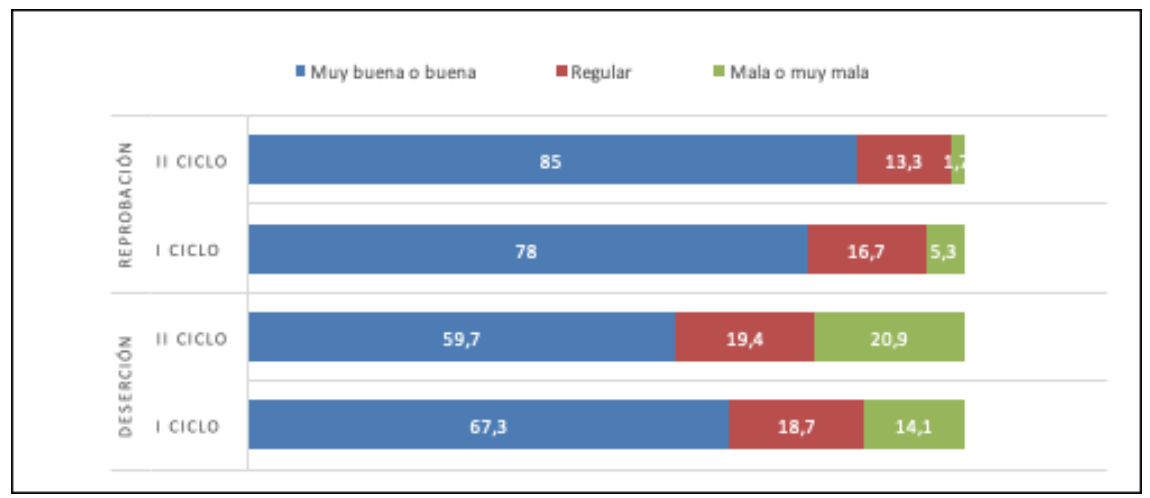

Fuente: Elaboración propia

En este curso se cuenta con el apoyo de horas consulta, en las cuales el estudiante puede aclarar sus dudas sobre ejercicios o teoría de una manera personalizada. Al consultarles sobre la asistencia a este espacio académico, se nota que no es aprovechado de la mejor manera. El 60,0\% de los estudiantes desertores indica que su asistencia es "mala" o "muy mala" mientras que en los estudiantes reprobados este porcentaje baja a un 50,0\%.

Después de analizar el comportamiento de la deserción y reprobados de los estudiantes desertores y reprobados del I y II ciclo, se observan tendencias similares en ambos grupos en aspectos familiares, de hábitos de estudios y compromiso con el curso.

En general, hace falta analizar esa percepción de los estudiantes, que no queda clara.

\section{ANÁLISIS DE LAS POSIBLES CAUSAS DE LA DESERCIÓN Y REPITENCIA SEGÚN SEXO, ÁREA DE PROCEDENCIA Y ESTRATO, DESDE LA PERSPECTIVA ESTUDIANTIL}

Un aspecto relevante en este trabajo consistía en determinar cuáles son las posibles causas de deserción y reprobación del curso de Matemática General, desde la percepción estudiantil. Por ello, se les consultó a los estudiantes su opinión por las posibles causas. En este apartado el análisis se hizo para las categorías de sexo, área de procedencia y estrato.

También se consideran a los estudiantes desertores y reprobados por aparte, según las categorías antes indicadas. Además, al consultarles a los estudiantes desertores del I ciclo lectivo cuáles consideraban que eran las posibles causas de deserción, mencionaron principalmente bajo rendimiento académico, conocimientos previos, tornarse nerviosos en las pruebas, malos hábitos de estudio y que el curso tenía muchos contenidos. Ante la consulta a los desertores del II ciclo sobre las posibles causas, ellos agregan la priorización por otras materias. Es decir, las causas de deserción coinciden en las dos poblaciones.

En la Figura 4, se presentan las causas de deserción por género. Se puede observar que el "bajo rendimiento académico" es la principal causa en todos los casos. En un segundo plano están los conocimientos previos adquiridos en secundaria, con un porcentaje cercano al 50,0\%, para hombres y mujeres. En el caso de los hombres en el II ciclo, mencionan como una de las principales los problemas de adaptación a la vida universitaria.

Para la reprobación, la principal causa son los conocimientos previos para los hombres en ambos ciclos. Las mujeres mencionan que la principal causa en el I ciclo son los conocimientos previos y en el II ciclo consideran que es el nerviosismo a la hora de realizar los exámenes. En la Tabla 13, se detallan las otras causas que mencionan, tanto hombres como mujeres, como las principales para la reprobación. Se nota también cómo los conocimientos previos son un motivo de reprobación para la mayoría de hombres y mujeres en ambos ciclos. Además, ambos sexos consideren el nerviosismo a la hora de realizar los exámenes como un motivo de la reprobación. 
Figura 4. Distribución porcentual de las causas de deserción por ciclo, según sexo, de los estudiantes del curso Matemática General, 2017

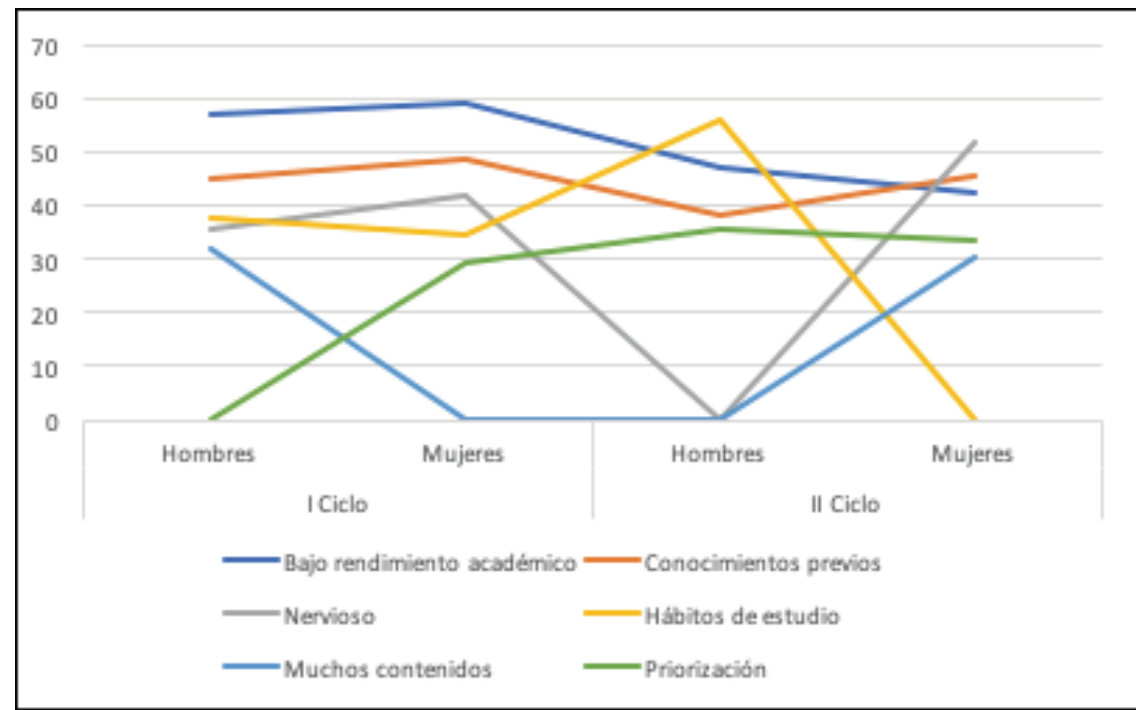

Fuente: Elaboración propia

Tabla 13. Distribución porcentual de las causas de reprobación por ciclo, según sexo, de los estudiantes del curso Matemática General, 2017

\begin{tabular}{lcccc}
\hline \multirow{2}{*}{ Causa de reprobación general } & \multicolumn{2}{c}{ I Ciclo } & \multicolumn{2}{c}{ II Ciclo } \\
\cline { 2 - 5 } & Hombres & Mujeres & Hombres & Mujeres \\
\hline Conocimientos previos & 64,4 & 72,7 & 70,0 & 54,3 \\
\hline Nervioso & 59,3 & 70,9 & 60,0 & 64,3 \\
\hline Priorización & 47,4 & 41,8 & 32,0 & 35,7 \\
\hline Hábitos de estudio & 50,8 & 34,5 & 56,0 & 30,0 \\
\hline Muchos contenidos & - & 32,7 & - & 38,6 \\
\hline Problemas de adaptación & - & - & 42,0 & 30,0 \\
\hline Poca dedicación & 56,0 & - & - & - \\
\hline Muchos cursos matriculados & - & 34,5 & - & - \\
\hline
\end{tabular}

Fuente: Elaboración propia

Costa Rica está dividida en dos áreas de desarrollo, la GAM y NGAM. La GAM es la región más urbanizada, poblada y económicamente activa del país. Al consultarles a los estudiantes sobre las causas de deserción por áreas, los estudiantes de la GAM aluden al bajo rendimiento académico, conocimientos previos, nerviosismo en las pruebas, malos hábitos de estudio y muchos contenidos. Mientras que los del NGAM señalan bajo rendimiento académico, conocimientos previos, nerviosismo durante las pruebas, malos hábitos de estudio y priorización a otras materias.

Respecto a la zona de procedencia de los estudiantes, las principales causas de deserción se presentan en la Figura 5. Las causas bajo rendimiento académico, nerviosismo y hábitos de estudios se mantienen como las principales causas de deserción tanto en el GAM como en el NGAM, en el I y en el II ciclos. Sin embargo, los estudiantes del I ciclo de la GAM 
indican que la cantidad numerosa de contenidos del curso es otra razón para considerar.
Por su parte, la causa "poca dedicación" también es mencionada por los estudiantes en el II ciclo lectivo, con un $35,0 \%$ y $30,0 \%$, respectivamente.

Figura 5. Distribución porcentual de las causas de deserción por ciclo, según área de procedencia, de los estudiantes del curso Matemática General, 2017

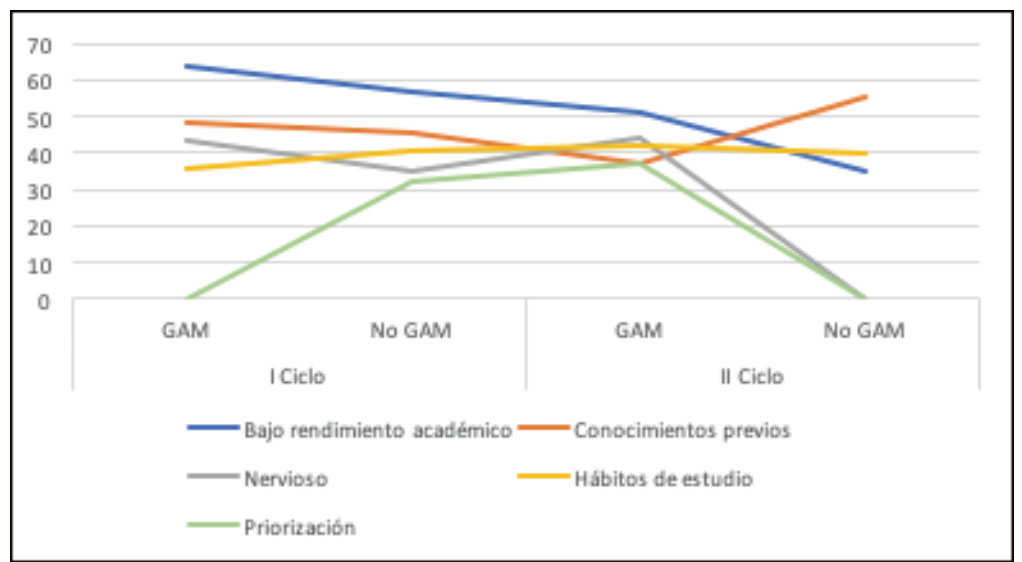

Fuente: Elaboración propia

Respecto a las causas de reprobación, en la Figura 6 se detallan "conocimientos previos" y "nerviosismo" como las principales que impactan en la reprobación, sin importar el área de procedencia. Los estudiantes del II ciclo de la GAM además, agregan, que los "muchos contenidos del curso" influyen en la reprobación.

Figura 6. Distribución porcentual de las causas de reprobación por ciclo, según área de procedencia, de los estudiantes del curso Matemática General, 2017

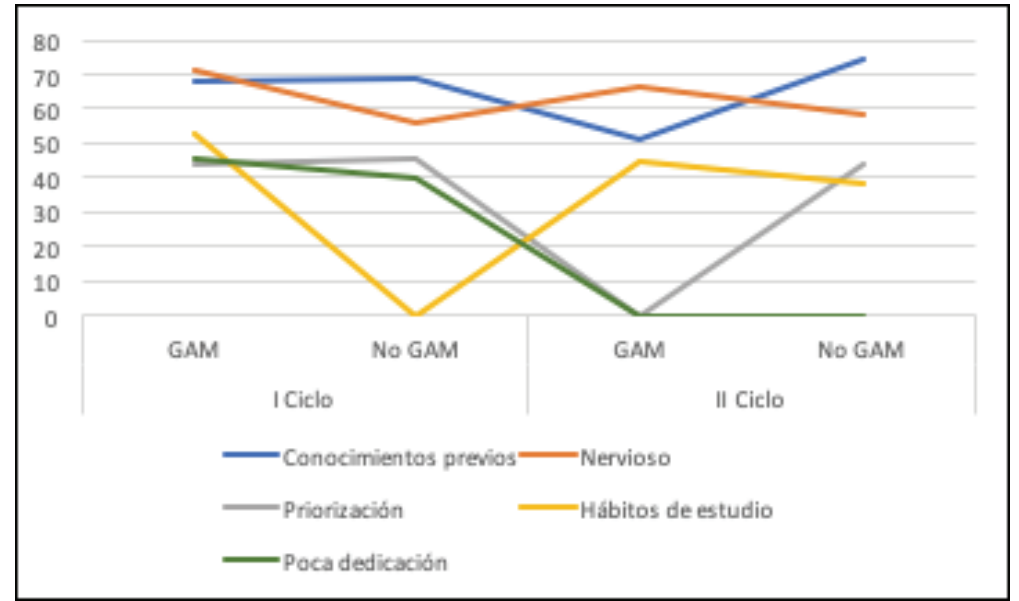

Fuente: Elaboración propia

Ante la consulta para conocer las causas de deserción por estratos, los del estrato I señalan que las principales causas de deserción son bajo rendimiento académico, priorización por otras materias, llegar 
tarde a clase, poca dedicación al curso y malos hábitos de estudio. Por su parte, los estudiantes del estrato II, indican que son bajo rendimiento académico, conocimientos previos, hábitos de estudio, se ponía nervioso en las pruebas y poca dedicación al curso. Los estudiantes del estrato III, nombran que se trata de los conocimientos previos, bajo rendimiento académico, nerviosismo en las pruebas, hábitos de estu- dio y la priorización. Como puede verse, las causas son iguales sin importar el estrato.

Respecto a la variable estrato, el bajo rendimiento académico es considerado el principal factor para la deserción independientemente del ciclo; en segundo lugar, pero en el estrato I, se ubica la priorización a otros cursos; el nerviosismo aparece como una opción, pero solo para el estrato II en el II ciclo.

Tabla 14. Distribución porcentual de las causas de deserción por ciclo, según estrato, de los estudiantes del curso Matemática General, 2017

\begin{tabular}{lcccc}
\hline \multirow{2}{*}{ Causa de deserción general } & \multicolumn{3}{c}{ I Ciclo } & II Ciclo \\
\cline { 2 - 5 } & Estrato I & Estrato II & Estrato III & Estrato II \\
\hline Conocimientos previos & - & 46,6 & 64,1 & 44,8 \\
\hline Nervioso & - & 40,9 & 33,3 & 48,3 \\
\hline Priorización & 31,8 & - & 25,6 & 41,4 \\
\hline Hábitos de estudio & 27,3 & 41,5 & 28,2 & 44,8 \\
\hline Poca dedicación & 27,3 & 32,4 & - & - \\
\hline Bajo rendimiento académico & 59,1 & 61,4 & 48,7 & 48,3 \\
\hline Llegar a clases & 27,3 & - & - & - \\
\hline
\end{tabular}

Fuente: Elaboración propia

En cuanto a las causas de reprobación por estratos, la información se observa en la Figura 7, en la cual se muestra que dentro de los principales motivos que indican los estudiantes están el nerviosismo y los co- nocimientos previos, independientemente del estrato y del ciclo donde se encuentren.

Figura 7. Distribución porcentual de las causas de reprobación por ciclo, según estrato, de los estudiantes del curso Matemática General, 2017

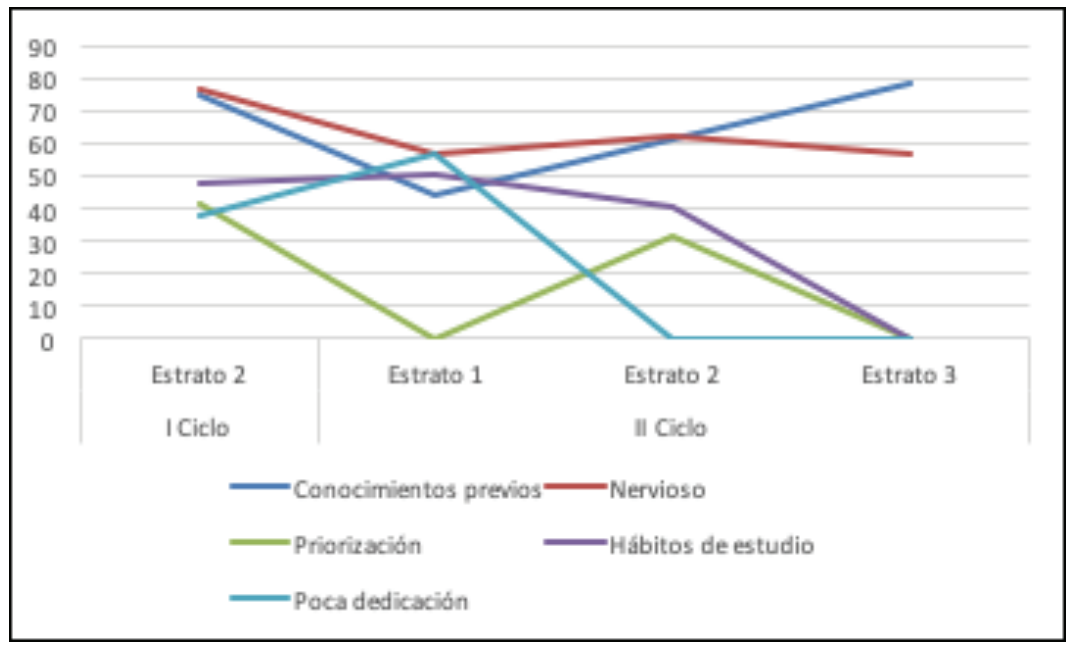

Fuente: Elaboración propia 
Los datos obtenidos en este trabajo son un incentivo para promover investigaciones futuras que analicen la problemática de la deserción en el curso de Matemática General, pues el abandono del curso trae consigo una mayor demanda de este en los ciclos lectivos posteriores, lo cual tiene implicaciones en el avance de los estudiantes en el plan de estudios y consecuencias económicas y académicas. Adicional a esta problemática, se presenta la reprobación en el curso.

Aspectos como el género, la edad, zonas de procedencia, tipo de institución de egreso de secundaria, carrera que cursa, situación laboral, entre otros, surgen como elementos que podrían tener relación, directa o indirecta, con la problemática de la deserción y reprobación.

Los conocimientos previos, bajo rendimiento académico en las pruebas parciales, factores emocionales asociados a las pruebas (nerviosismo) e inadecuados hábitos de estudio surgen como causas mencionadas por el estudiantado que provocan tanto deserción como reprobación.

A partir de lo anterior, se evidencia la necesidad de plantear estrategias para la atención integral de los estudiantes, especialmente los de primer ingreso a la universidad, que involucren no solo aspectos de formación académica sino el desarrollo de habilidades y capacidades para afrontar los estudios universitarios en matemática, en particular, y de sus respectivas carreras, en general.

\section{DISCUSIONES FINALES}

Con base en la investigación realizada, a pesar de que el diseño muestral no permite realizar inferencias, se hace un resumen de los principales resultados:

1. Existe un alto porcentaje de deserción en el curso de Matemática General independientemente del ciclo.

2. Más del 50,0\% de los hombres desertaron en el I y II ciclo del año 2017.

3. El mayor porcentaje de aprobación se presenta en las mujeres en ambos ciclos.
4. El porcentaje de reprobación es mayor en las mujeres que en los hombres.

5. En el II ciclo los porcentajes de reprobación y deserción disminuyen y aumentan los niveles de aprobación, posiblemente asociados a que para el II ciclo se da un proceso de aprendizaje o de adaptación a la vida universitaria.

6. La población que matricula el curso de Matemática General es relativamente joven (la mayoría tiene menos de 21 años) y más del 50,0\% de ese grupo (21 años o más) deserta del curso.

7. La mayoría de los estudiantes proviene de colegios públicos y presenta menor porcentaje de aprobación y mayor porcentaje de reprobación, en comparación con los estudiantes de colegios privados o subvencionados.

8. La carrera de Ingeniería en gestión ambiental es la que tiene menos deserción en ambos ciclos.

9. Una gran mayoría de estudiantes de Matemática General cursa la carrera que seleccionaron como primera opción.

10. Los resultados encontrados apoyan la teoría que relaciona las variables de deserción y situación laboral.

11. Los estudiantes que deben laborar cierta cantidad de horas a la semana, tienen una alta deserción en el curso de Matemática General.

12. Según la clasificación de los estratos que define la Universidad Nacional, la mayoría de los estudiantes se ubican en el número 2.

13. Se presenta la siguiente relación según estratos: estrato I, mayor aprobación; estrato II, mayor reprobación; estrato III, mayor deserción.

Con respecto a la muestra consultada para establecer posibles causas de deserción y reprobación, se puede concluir lo siguiente:

1. La mayoría de los estudiantes desertores cursaba el primer año de carrera, lo cual concuerda con la ubicación del curso en las mallas curriculares. Sin embargo, es importe señalar que se presentaron casos de estudiantes de segundo, tercero y cuarto años, lo cual podría ser un indicador que este no es un curso requisito para avanzar en su carrera. 
2. La mayoría de los estudiantes desertores y reprobados se perciben como "muy buen estudiante" y "buen estudiante".

3. La mayoría consideraba que el rendimiento académico en secundaria en matemática era "muy bueno" o "bueno".

4. La mayoría de los estudiantes desertores y reprobados concuerda en que los conocimientos de secundaria no son suficientes para aprobar el curso de Matemática General.

5. La mayoría de estudiantes no estudia a diario este curso y relata que la cantidad de horas de estudio es inferior a la recomendada por el programa del curso.

6. La mayoría de los estudiantes comenta que asiste regularmente a las clases; sin embargo, se presenta un desaprovechamiento de las horas de consulta.

7. Las causas de deserción y reprobación que priorizan los estudiantes, sin importar el género, área geográfica y estrato, son el bajo rendimiento académico, conocimientos previos y hábitos de estudio. Ante esto, consideramos que se debe formular una política institucional que permita analizar a los estudiantes desertores y reprobados y así atender a cada grupo, dependiendo de sus características particulares, e involucrar en el proceso a otras instancias de la universidad.

8. La causa del nerviosismo en las pruebas resulta importante, por lo se debe ahondar en sus causas y hacer un estudio especializado.

9. Se recomienda también la implementación de un curso de nivelación que permita a los estudiantes reforzar contenidos estudiados durante la secundaria.

10. Se debe realizar más investigación de aula para determinar otras causas e implicación de la deserción y reprobación, con el fin de medir el impacto de los fenómenos en términos de económicos y sociales, entre otros.

Los resultados de esta investigación permitieron evidenciar que la principal problemática del curso de Matemática General es la deserción, seguida por la reprobación. Además, permitió la identificación de ciertas características sobre el tipo de población con estas problemáticas, para generar así diversas estrategias para la identificación y atención oportuna.
Los datos mostrados en este artículo pretenden aportar al estudio de estas problemáticas a nivel universitario, y que sirvan de base para estudios futuros en el tema y en la profundización de los factores asociados.

\section{REFERENCIAS BIBLIOGRÁFICAS}

Abarca, A. y Sánchez, M. A. (2005). La deserción estudiantil en la educación superior: el caso de la Universidad de Costa Rica. Actualidades Investigativas en Educación, 5, 1-22. Recuperado de https:// www.redalyc.org/articulo.oa?id=44759911

Canales, A. y De los Ríos, D. (2018). Factores explicativos de la deserción universitaria. Calidad en la Educación, 26, 173-201. Recuperado de https:// www.researchgate.net/publication/26522274_ Factores_explicativos_de_la_desercion_universitaria

Centro Interuniversitario de Desarrollo, CINDA. (2006). Repitencia y deserción universitaria en América Latina. Santiago, Chile: Colección Gestión Universitaria-IESALC. Recuperado de https:// cinda.cl/wp-content/uploads/2006/05/repitenciay-desercion-universitaria-en-latinoamerica.pdf.

Chaves, E., Castillo, M. y Gamboa, R. (2008). Correlación entre el examen de admisión y el rendimiento en el primer año de la carrera Enseñanza de la Matemática en la UNA. Revista Electrónica Educare, 12(2), 65-80. Recuperado de https://doi. org/10.15359/ree.12-2.4.

Corral, V. y Díaz, X. (2009). Factores Asociados a la Reprobación de los Estudiantes de la Universidad de Sonora. Memorias del X Congreso Nacional de Investigación Educativa, México. Recuperado de http://www.comie.org.mx/congreso/ memoriaelectronica/v10/pdf/area_tematica_16/ ponencias/0910-F.pdf.

De Marchis, G. P. (2012). La validez externa de las encuestas en la" web". Amenazas y su control. Estudios sobre el mensaje periodístico, 18, 263-272. Recuperado de https://revistas.ucm.es/index.php/ ESMP/article/viewFile/40980/39231. 
Departamento de Registro Universidad Nacional (2019). Estadísticas Estudiantiles de la Universidad Nacional de Costa Rica. Recuperado de https:// www.registro.una.ac.cr/see/index.php/rendimiento.

González, L. (2007). Repitencia y deserción universitaria en América Latina. En UNESCO-IESALC (ed.), Informe sobre la educación superior en América Latina y el Caribe 2000-2005: La metamorfosis de la educación superior, 156-168. Recuperado de http://www.alfaguia.org/alfaguiav2/ files/1319033299_01.pdf.

González, L., Uribe, D. y González, S. (2005). Estudio sobre la repitencia y deserción en la educación superior chilena. Estudio IESALC, Chile. Recuperado de https://unesdoc.unesco.org/ark:/48223/ pf0000140087.

Lentini, M. L., Martínez, I. Z., Lentini, M. C., Tarifa, G. E. y Crespo, S. H. (2003). Repitencia de alumnos en los primeros cursos de Matemática: La importancia de su erradicación. Ponencia presentada en el Congreso Latinoamericano de Educación Superior en el Siglo XXI, San Luis, Argentina. Recuperado de bd.unsl.edu.ar > download4.

Páramo, G. y Correa, C. (1999). Deserción estudiantil universitaria. Conceptualización. Revista Universidad EAFIT, 35(114), 65-78. Recuperado de http:// publicaciones.eafit.edu.co/index.php/revista-universidad-eafit/article/view/1075/967.

Pascua-Cantero, P. (2016). Factors Related to Dropping Out in the Freshman and Sophomore Years in the Career of Teaching Mathematics at Universidad Nacional de Costa Rica. Revista Electrónica Educare, 20(1), 1-23. Recuperado de https://doi. org/10.15359/ree.20-1.5.

Rodríguez, I. (2017). La calidad de la educación superior y la reestructuración del programa de tutoría. RIDE. Revista Iberoamericana para la Investigación y el Desarrollo Educativo, 8(15), 135-154. Recuperado de http://www.scielo.org.mx/scielo.php?script=sci_arttext\&pid =S2007-74672017000200135.
Rodríguez, A. y Vindas, M. (2005). La deserción estudiantil en la educación superior: el caso de la Universidad de Costa Rica. Actualidades investigativas en educación, 5. Recuperado de https://revistas. ucr.ac.cr/index.php/aie/article/view/9186.

Salazar, C. C. y Espinoza, C. M. R. (2017). Principales causas de reprobación de alumnos de los grupos de quinto semestre grupo seis y ocho de la escuela preparatoria número tres. (Capítulo I Antecedentes). Con-Ciencia Boletín Científico de la Escuela Preparatoria. 3(4). Recuperado de https://www. uaeh.edu.mx/scige/boletin/prepa3/n7/p1.html.

Torres, J., Acevedo, D. y Gallo, L. (2015). Causas y consecuencias de la deserción y repitencia escolar: una visión general en el contexto Latinoamericano. Cultura Educación y Sociedad 6(2), 157-187. Recuperado de https://pdfs.semanticscholar.org/4a 35/45b9900ab422002e3715e17332167dfb4163. pdf.

Viale, H. (2015). Una aproximación teórica a la deserción estudiantil universitaria. Revista Digital de Investigación en Docencia Universitaria (RIDU), 8(1), 59-75. Recuperado de https://dialnet.unirioja. es/servlet/articulo?codigo $=4898826$.

Zamora-Araya, J. A. y Villalobos-Madrigal, F. J. (2018). Factors associated with dropping out of the Bachelor's Degree program in Mathematics Teaching at the Universidad Nacional de Costa Rica (UNA): Evidence from the 2016 Student Cohort. Revista Uniciencia. 32(2), 111-126. Recuperado de http://dx.doi.org/10.15359/ru.32-2.8. 